\title{
Creating a Cleaner Informing System to Increase the Percentage of Clean Beds in the Post Cesarean Ward at Nyagatare Hospital, Rwanda
}

\author{
Vital Kayiranga1, Angele Musabyimana², Eva Adomako³, April Budd ${ }^{3}$, \\ Philippe Ngabire Nkunda ${ }^{1}$, Rex Wong 3 \\ ${ }^{1}$ Nyagatare Hospital, Nyagatare, Rwanda \\ ${ }^{2}$ School of Public Health, University of Rwanda, Kigali, Rwanda \\ ${ }^{3}$ Yale Global Health Leadership Institute, Yale University, New Haven, CT, USA \\ Email: rex.wong@yale.edu
}

Received 21 June 2016; accepted 2 August 2016; published 5 August 2016

Copyright (C) 2016 by authors and Scientific Research Publishing Inc.

This work is licensed under the Creative Commons Attribution International License (CC BY).

http://creativecommons.org/licenses/by/4.0/

(c) (i) Open Access

\begin{abstract}
Hospital beds are one of the most frequently used non-invasive equipment in the clinical area and have a high risk of transmitting hospital acquired infections. However, they are usually not cleaned effectively. Accordingly, we conducted this quality improvement project to improve the bed cleaning process in a rural district hospital in Rwanda. A pre-post intervention study was conducted from in April 2015 to February 2016. The beds in the maternity unit were observed after patient was discharged. We implemented an alert system included using a signage system to help inform cleaners to clean the beds. Guidelines for the new system were created and trainings were provided. The intervention significantly increased the percentage of clean beds from $44 \%$ pre-intervention to $90 \%$ post-intervention $(P<0.001) .93 \%$ of staff followed the new labelling system. By focusing on system and process change, this quality improvement project successfully increased the percentage of clean beds without additional financial investment.
\end{abstract}

\section{Keywords}

Hospital Acquired Infection, Bed Cleaning, Quality Improvement, Resource-Limited Setting

\section{Introduction}

Hospital beds are one of the most frequently used non-invasive reusable communal equipment in the clinical

How to cite this paper: Kayiranga, V., Musabyimana, A., Adomako, E., Budd, A., Nkunda, P.N. and Wong, R. (2016) Creating a Cleaner Informing System to Increase the Percentage of Clean Beds in the Post Cesarean Ward at Nyagatare Hospital, Rwanda. Journal of Service Science and Management, 9, 342-346. http://dx.doi.org/10.4236/jssm.2016.94039 
area [1]-[3]. Due to their frequent use in clinical areas, particularly in those that have constant high turnover of patients, hospital beds have a high risk of transmitting hospital acquired infections to patients and staff [1]-[6]. Hospital beds, including the frames, mattresses and the linen may become source of infection [4] [7]-[9]. However, in a busy hospital environment, hospital beds are usually not cleaned effectively [3].

Hospital acquired infections could affect patients negatively including increased length of stay and higher risks of developing complications. It also has negative financial impact as it incurs higher cost for patients as well as for hospitals. High incidence of hospital acquired infections can negatively affect patient satisfaction and eventually, patient and community confidence towards the hospital [3] [10]. Given the many negative impacts of hospital acquired infections on the patients and the hospital, proper infection prevention and control measures should be in place to ensure hospital beds which are clean to prevent cross infection.

However, there is no scientific and conclusive approach recommended for bed cleaning. Many studies agree that beds should be decontaminated between each patient use, after soiling and at regular intervals, whether in use or not and as part of regular infection control practice [11] [12].

Other literature has suggested guidelines for cleaning and disinfecting hospital beds [7] [10] [13] [14]. Most of the suggested guidelines however are best practices. There is insufficient scientific evidence and research around bed cleaning [14] [15]. The generally agreed upon cleaning approach is to clean systematically, from the top or furthest point away of the equipment, or the area that connects with the patient that should be cleaned first [7] [8] [10] [11] [13] [16]-[18]. Since there is no scientific and conclusive approach recommended for bed cleaning, different methods are used to ensure beds provided to patients are clean. Some of the methods used include setting up documentation for the person cleaning the bed, creating rigid cleaning schedules, spot auditing and proper labeling to indicate when a bed has undergone decontamination [12] [13] [19]. There is a general lack of published papers documenting a systematic approach to enhance hospital bed cleaning. Accordingly, we conducted this quality improvement project to improve the bed cleaning process in a district hospital in Rwanda.

\section{Methodology}

\subsection{Setting}

The study was conducted in Nyagatare district hospital in the Eastern province of Rwanda. It is a 200 bed hospital receiving between 3500 and 4000 patients per month. The Maternity Department in the Nyagatare District Hospital has 38 beds with an average bed occupancy rate of $99.7 \%$.

Before our intervention, there were no cleaners assigned specifically to the cesarean ward. Unless they had been present in the ward when patients were being discharged, cleaners who came to clean did not know whether an empty bed was dirty or clean. The nurse who discharged the patient was responsible for finding a cleaner. Occasionally, nurses would forget or become too busy to inform the cleaners. As a result, sometimes when a new patient was admitted, the nurse would be unclear of the bed's cleanliness; potentially admitting the patient into a contaminated bed. According to an observation study we conducted in April 2015, 29 times out of the 40 discharges (72\%), the cleaners did not show up to clean the bed. Out of the 29 times when cleaners did not show up, 22 times (76\%) were because nurses forgot to inform cleaners about the discharge.

\subsection{Study Design and Sample}

This study used a pre-post intervention study design. The pre intervention period began in April 2015. During the pre-intervention period, we conducted a study to measure the magnitude of the problem, we then conducted a root cause analysis that allowed us to generate a strategy to address the problem. Implementation started in February 2016 and evaluation was conducted immediately after official implementation.

To measure the percentage of clean beds in the ward, the team, including the director of nursing, nurse in charge of maternity, and the environmental health officer, conducted an observation study in April 2015. The study included spot checks to see if beds were clean before a new patient was admitted. The team spent two weeks in the cesarean ward, spot checked the beds both in the morning and in the afternoon, trying to match the time when patients were discharged from the ward. A hospital bed was considered not clean when any one of the five criteria existed: (1) disinfectant was not used; (2) presence of blood; (3) presence of fecal matter and urine; (4) damaged mattress and/or bed sheet; (5) presence of body fluids. The same assessment procedures were 
repeated in the post-intervention period to collect evaluation data for comparison.

\subsection{Intervention}

The team implemented a new alert system for bed cleaning when patients are discharged. The new alert system included using a signage system to label beds as "dirty" when patients were discharged. Once the nurse finished the discharge process and the patient had left the bed, the nurse would put a paper sign saying "dirty" on the bed. The sign served multiple purposes: 1) As a reminder for the nurse to inform the cleaner. The nurse in charge of the discharge was responsible for informing the cleaner immediately after the bed was empty. However, they would sometimes forget to inform because they were too busy. With a "dirty" sign on the bed, every time the nurse saw the sign, he/she was reminded of contacting the cleaner. 2) As a visual informing system for cleaners. If the nurses forget to inform the cleaners, when the cleaners saw the "dirty" sign, they knew the bed needed to be cleaned, even if they hadn't been informed by the nurses. 3) As a warning sign. The "dirty” sign warned nurses not to put any new patient in the bed, as it had not been cleaned. When a new patient was admitted, the "dirty" sign would inform the admitting nurse that the bed had not been cleaned, thus avoiding miscommunication and putting patients in dirty beds.

The guidelines for the new system was drafted and approved in December 2015. Trainings were arranged for all staff including nurses and cleaners of the post-cesarean ward, in January 2016. The new system officially started in February 2016. The project was approved by the senior management team of the hospital.

\subsection{Measure}

The percentages of beds cleaned in the pre intervention and post intervention periods were used to evaluate the success of the intervention. The percentage of bed using label system in the post intervention was also recorded.

\subsection{Data Analysis}

The percentage of bed cleaned in the pre intervention and post intervention period were compared and analyzed using Fisher Exact Test, using SPSS v.21, with P-value set at $\leq 0.05$.

\section{Results}

In the pre intervention period, there were 18 patients discharged from the post-cesarean ward. Eight out of the 18 beds (44\%) were clean. In the post intervention period, there were 41 patients discharged from the post-cesarean ward. Thirty nine out of the 41 beds $(90 \%)$ were cleaned. There is significant increase in the percentage of clean bed from $44 \%$ pre intervention to $90 \%$ post intervention, with $\mathrm{P}<0.001$. The most important contributing factor was the reduction of fecal matter found in bed. In our pre-intervention, we found $22 \%$ of beds (4 out of 18) had fecal matter in them whereas, it was significantly reduced to $2 \%$ (1 out of 41 ) after the intervention implementation $(\mathrm{P}=0.021)$. The post-intervention result showed $93 \%$ of staff followed the labelling system (Table 1).

Other observed elements such as using disinfectant and the presence of blood or other body fluids in beds, did not show significant statistical difference despite the fact that they all showed reduction from per-intervention to post-intervention.

\section{Discussion}

This quality improvement project was designed to address the low percentage of clean beds in the caesarean ward of Nyagatare hospital. The results showed that the intervention increased the percentage of clean beds. We also found that compliance to the new system was very high. The three instances of noncompliance to the labelling system was a result of new untrained nurses working in the cesarean ward. The reduction of fecal matter found in beds was most substantial. Fecal matter was probably the most visible and frequent item found in beds. By simply cleaning the bed and changing the bed sheet, the prevalence was reduced significantly.

The project did not aim at improving cleaning methods or changing mattress, but rather, focus on the awareness of cleaners and nurses that the beds needed to be cleaned. Accordingly, we did not expect significant improvement in the percentage of use of disinfectant or the percentage of clean mattress. The project did not 
Table 1. Pre-and post-intervention result comparison.

\begin{tabular}{ccccc}
\hline & Pre-intervention & Post-intervention & Change & P value \\
\hline Sample size $(\mathrm{N})$ & 18 & 41 & - & - \\
Bed labelled & - & $38(93 \%)$ & $15 \%$ & 0.119 \\
Not using disinfect & $4(22 \%)$ & $3(7 \%)$ & $11 \%$ & 0.089 \\
Blood found in bed & $2(11 \%)$ & $0(0 \%)$ & $20 \%$ & $0.021^{*}$ \\
Fecal found in bed & $4(22 \%)$ & $1(2 \%)$ & $15 \%$ & 0.08 \\
Mattress dirty & $3(17 \%)$ & $1(2 \%)$ & $4 \%$ & 0.521 \\
Fluid found in bed & $1(6 \%)$ & $3(2 \%)$ & $46 \%$ & $<0.001^{*}$
\end{tabular}

*Statistical significant at $\mathrm{P} \leq 0.05$.

significantly reduce blood or fluid found in beds. A possible explanation was that the percentage of blood and body fluid found in beds were already low in pre-intervention, so significant reductions could not be seen. However, further investigation and longer follow up should be conducted to understand the reason.

The success of this project relied on a few things: the support from senior management team (SMT), a detail root cause analysis, a simple and effective intervention, the involvement of a multidisciplinary team, and incorporating feedback from the staff. Support from senior management team was important not only because they had to approve the project and provide the needed materials, but also, their leadership and encouragement were sources of motivation for concerned staff. It was also easier to communicate our objective and new process to the staff when the SMT showed their support for the project. A detailed root cause analysis allowed us to understand the real issues of the process and to create an effective intervention. Without understanding the real root caused behind beds not being cleaned, our intervention could have been focused on relatively minor causes, and created less satisfactory results.

This simple, effective and easy to use bed labeling system did not cause the hospital any investment or require technical training or sophisticated equipment. The sign system provided multiple functions as to remind nurses to inform cleaners, to inform cleaners to clean beds, and to warn nurses not to put patients in beds that had not been cleaned. Involving all stakeholders at the early phase was helpful in gaining their buy-in. It also allowed us to gain thorough understanding of the cleaning process from different perspectives. We collected and incorporated comments from the front line staff who used the new process. For example, we received comments from nurses in the unit saying sometimes they could not remember if the bed was actually cleaned or if the nurse had forgotten to place the dirty label. Sometimes, the cleaners also did forget to remove the dirty label after cleaning. They suggested a second "cleaned" label be added to improve the process, which we did.

Although the results showed significant improvement in bed cleaning, the implementation was met with some challenges. Initially, some cleaners and nurses were skeptical about the new process. Such concerns were discussed with the staff to ensure their understanding. We also provided them the initial evaluation results to show them the positive changes the new system had brought on. Showing them the success helped us minimize their resistance and cement their commitment to continue the project.

This project has some limitations. It focused on the alert system and did not address the quality and method of cleaning. Other quality improvement projects should be conducted to address these issues. Longer term follow up is needed to ensure sustainability of the project. Regular refresher trainings should be conducted to ensure new nurses and cleaners working in the unit also understand and follow the system.

This study was conducted to increase the percentage of clean beds by creating a bed cleaning alert system after every discharge. Based on evidence and data, the project created an intervention focused on changing the system which did not cost the hospital any additional financial investment. The intervention successfully increased the bed cleaning in the cesarean ward of Nyagatare Hospital. 


\section{References}

[1] Creamer, E. and Humphreys, H. (2008) The Contribution of Beds to Healthcare-Associated Infection: The Importance of Adequate Decontamination. Journal of Hospital Infection, 69, 8-23. http://dx.doi.org/10.1016/j.jhin.2008.01.014

[2] Young, J.M., Naqvi, M. and Richards, L. (2005) Microbial Contamination of Hospital Bed Handsets. American Journal of Infection Control, 33, 170-174. http://dx.doi.org/10.1016/j.ajic.2004.11.005

[3] O’Connor, H. (2000) Contaminating Beds and Mattresses. Nursing Times, 96, 4-5.

[4] Ayliffe, G.A., et al. (1993) Chemical Disinfection in Hospitals. Public Health Laboratory Service, London.

[5] Rutala, W.A. and Weber, D.J. (2008) Healthcare Infection Control Practices Advisory Committee. Guideline for Disinfection and Sterilization in Healthcare Facilities. Centers for Disease Control and Prevention, Atlanta.

[6] Schabrun, S. and Chipchase, L. (2006) Healthcare Equipment as a Source of Nosocomial Infection: A Systematic Review. Journal of Hospital Infection, 63, 239-245. http://dx.doi.org/10.1016/j.jhin.2005.10.013

[7] Krankenhaus and Praxishygiene Working Group (2005) Hygiene in Hospital and Practice. 3rd Revised and Edited Edition, Weisbaden, 157-161.

[8] Vary, J. (2014) Environmental Cleaning in the Emergency Department. College of Saint Benedict/Saint John University.

[9] McGoldrick, M. (2009) Cleaning and Disinfection of Patients Care Equipment Used in the Home Setting. Caring: National Association for Home Care Magazine, 28, 34-39.

[10] Patel, S. (2005) Minimizing Cross Infection and Risks Associated with Beds and Mattresses. Nursing Times, 101, 5253.

[11] National Patient Safety Agency (2009) The Revised Healthcare Cleaning Manual. NPSA, London.

[12] Healthcare Associated Infection Task Force (2004) The NHS Scotland Code of Practice for the Local Management of Hygiene and Healthcare Associated Infection. Scottish Executive, Edinburgh.

[13] Standard Infection Control Literature Review: Management of Patient Care Equipment in the Hospital Setting Version 1.0. January 2012.

[14] Price, E.H. and Ayliff, G. (2008) Hot Hospitals and What Happened to Wash, Rinse and Dry? Recent Changes to Cleaning, Disinfection and Environmental Ventilation. Journal of Hospital Infection, 69, 89-91. http://dx.doi.org/10.1016/j.jhin.2008.01.009

[15] Dancer, S.J. (2009) The Role of Environmental Cleaning in the Control of Hospital-Acquired Infection. Journal of Hospital Infection, 73, 378-385. http://dx.doi.org/10.1016/j.jhin.2009.03.030

[16] Medicines and Healthcare Products Regulatory Agency (2006) Sterilization, Disinfection and Cleaning of Medical Equipment: Guidance on Decontamination from the Microbiology Advisory Committee (the MAC Manual): Part 3 Procedures. MHRA, London.

[17] Department of Health (2010) Clean Safe Care. High Impact Intervention: Cleaning and Decontamination. Department of Health, London.

[18] Rutile, W.A., Weber, D.J., HICPAC (2008) Guideline for Disinfection and Sterilization in Healthcare Facilities. CDC. www.cdc.gov/hicpac/pdf/guidelines/disinfection_Nov_2008.pdf

[19] Medicines and Healthcare Products Regulatory Agency (2005) Sterilization, Disinfection and Cleaning of Medical Equipment: Guidance on Decontamination from the Microbiology Advisory Committee (the MAC Manual): Part 2 Protocols. MHRA, London. 


\section{Submit or recommend next manuscript to SCIRP and we will provide best service for you:}

Accepting pre-submission inquiries through Email, Facebook, LinkedIn, Twitter, etc.

A wide selection of journals (inclusive of 9 subjects, more than 200 journals)

Providing 24-hour high-quality service

User-friendly online submission system

Fair and swift peer-review system

Efficient typesetting and proofreading procedure

Display of the result of downloads and visits, as well as the number of cited articles

Maximum dissemination of your research work

Submit your manuscript at: http://papersubmission.scirp.org/ 